\title{
Editorial: Use of Earth Observations for Actionable Decision Making in the Developing World
}

\author{
Niall P. Hanan ${ }^{1 *}$, Ashutosh S. Limaye ${ }^{2}$ and Daniel E. Irwin ${ }^{2}$ \\ ${ }^{1}$ Plant and Environmental Sciences Department, New Mexico State University, Las Cruces, NM, United States, ${ }^{2}$ National \\ Aeronautics and Space Administration (NASA), Marshall Space Flight Center, Huntsville, AL, United States
}

Keywords: Earth Observation, SERVIR, satellite data, applied research, sustainable development

Editorial on the Research Topic

Use of Earth Observations for Actionable Decision Making in the Developing World

\section{INTRODUCTION TO THE SPECIAL TOPIC}

The global community faces fundamental challenges related to natural resource management, particularly in low and middle income countries. Increasing populations and changing climate exacerbate the challenges of food security, water, and environmental management and sustainability faced by rural communities, cities, and governments alike. Sound environmental and risk management decisions must often be made with incomplete or inadequate information, particularly in the developing world. However, Earth Observations (EO) can bring objective data to developing-country land-users, land managers and policymakers by addressing key information gaps through consistent and repeatable observations (Zell et al., 2012) and through capacity development efforts (Kumar et al., 2020).

Technological innovations and proactive EO agendas developed by space agencies around the world are helping (Brown et al., 2013). In the United States, for example, the prioritization of key EO science and applications in the recent National Academies decadal survey (National Academies of Sciences, Engineering, and Medicine, 2019) will ensure continuity of orbiting sensors that measure critical Earth system parameters. Similarly, the European Space Agency's Copernicus program (Brachet, 2004) makes high quality and up to date satellite data freely available, and consequently the use of these data is expanding rapidly. There is an explosion of active, ongoing research in the US and in the developing world that can directly translate into working theories and operational systems for developing countries. Ensuring alignment between the research community and decision makers, and orienting the latest science from the United States to help solve local/regional problems, has been the primary objective of the NASA Applied Sciences Program (Friedl, 2017). The purpose of the articles in the Special Topic "Operational Earth Observation for Sustainable Development and Risk Reduction in the Developing World" is to focus on the challenges and solutions for use of $\mathrm{EO}$ data for actionable decision making around the world, particularly in developing countries.

SERVIR is a joint initiative of NASA and the U.S. Agency for International Development (USAID) which fosters applications of EO to help developing countries assess environmental conditions to improve planning and management interventions. Working with regional technical organizations ("regional hubs") around the world, SERVIR aims to improve resilience and sustainable resource management at local, national, and regional scales through the increased use of Earth Observations, Earth science, and technology. SERVIR hubs have regional mandates to work with national governments of their member countries, thus promoting strong pathways between 


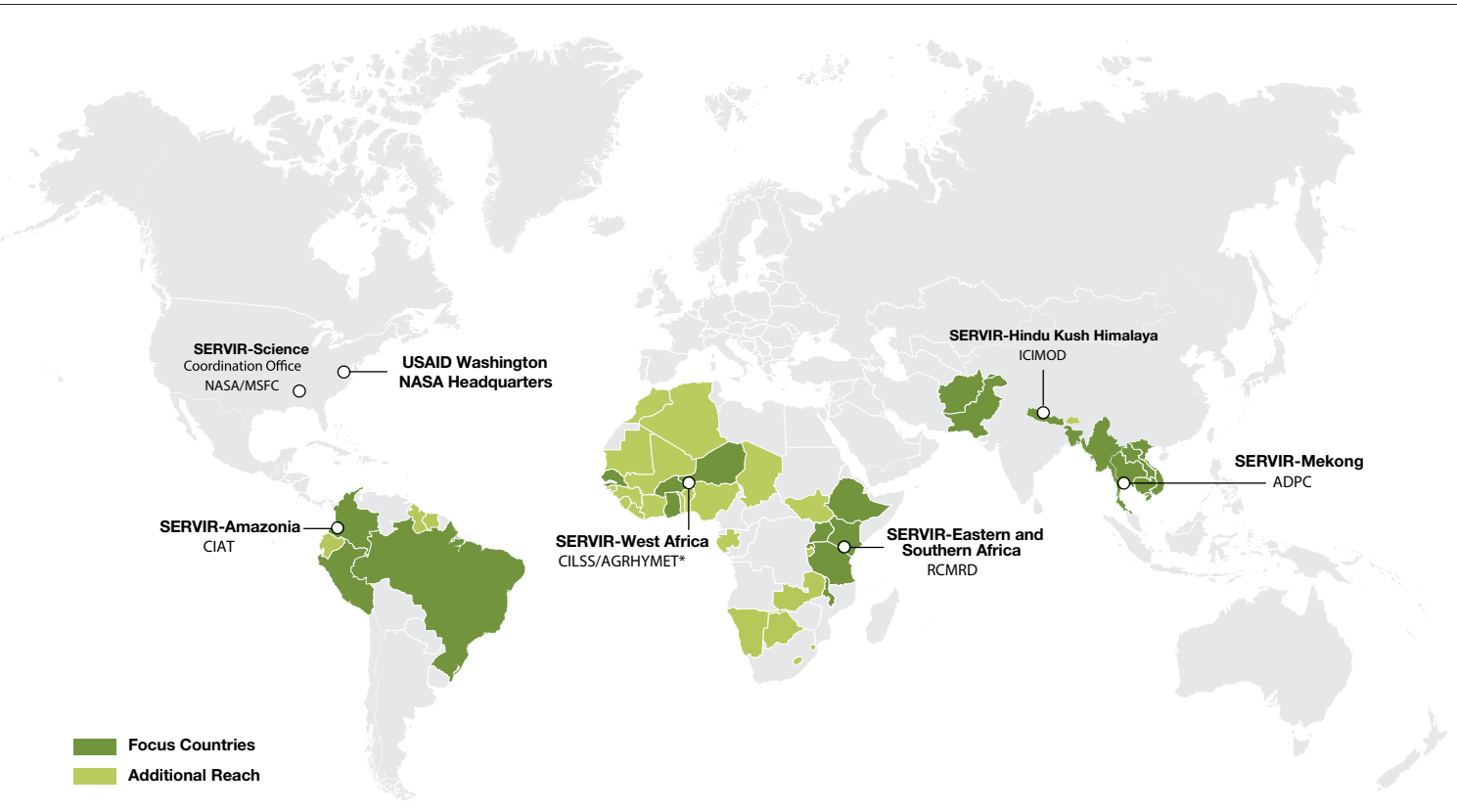

FIGURE 1 | Geographic regions and primary countries served by the five SERVIR regional hubs.

EO-based solutions and policy makers. SERVIR hubs are active in five regions (Figure 1), including Eastern and Southern Africa, the Hindu Kush Himalaya (HKH), lower Mekong, West Africa, and most recently in Amazonia. SERVIR focuses on four themes: land use, land cover, and ecosystems; food security and agriculture; water and related disasters; and weather and climate. For the land cover theme, SERVIR has ongoing activities in all regions, to strengthen the capacity of regional partners to link the latest science to promote sustainable use of natural resources. In agriculture, SERVIR promotes EO-based crop monitoring technologies to better analyze and predict food security conditions. Connecting satellite-derived rainfall and other datasets helps SERVIR improve the capacity of countries to forecast streamflow and identify regions where water-related disasters can occur. Weather and climate activities inform the other themes in ensuring the latest and robust availability of weather information, in addition to short- and long-term weather forecasts.

Articles in this Special Topic introduce a range of EO-based approaches for monitoring natural resources, risk, and disaster analysis and prediction in SERVIR regions of Africa, HimalayaHindu Kush, and Southeast Asia. These include the use of EO for hydrological monitoring, drought and flood prediction, land use, land cover and agricultural monitoring, and invasive species. Several papers focused on emerging remote sensing technologies with applications in less developed regions, while others focused on applications and stakeholders from local (village) scale to national, regional, and global scales.

Two papers focus explicitly on the critical phases of stakeholder engagement during design, development, and implementation of EO applications to enhance relevance, use and impact. The paper by Thapa et al. documents the steps involved in engaging relevant communities to define their needs and design appropriate EO applications. Saah et al. provide a specific example of the user engagement process during development of land cover mapping tools for diverse users in the Mekong Basin and Hindu Kush-Himalaya regions.

Several papers focused on use of EO as inputs for hydrological modeling applications. Purdy et al. focused on the use of EO for analysis of groundwater dynamics and agricultural sustainability in Bangladesh. Nelson et al. and Alcantara et al. demonstrate the utility of cloud-based hydrological modeling systems for stream-flow and flood prediction, with a focus on the transfer of model outputs into actionable information for regional and local decision makers. Sikder et al. analyze the impact of EO inputs, land surface model, and river routing schemes on riverflow predictions in the critical Ganges-Brahmaputra and Mekong river basins of South and Southeast Asia. Oddo and Bolten demonstrate that the integration of satellite data into a flood modeling system for the city of Bangkok, Thailand can be of immense value in potential lives and property saved through shorter emergency response times. Phongsapan et al. explore the utility of satellite data and improved hydrological forecasting for flood risk reduction in Myanmar.

An analysis by Spruce et al. for the Lower Mekong Basin revisits the difficult process of land use and land cover (LCLU) classification, particularly in the context of temporal analysis of change, where classification errors in individual time-periods can be compounded in change analysis. The LCLU maps made available for the region are important for a variety of EO applications, including hydrological and agricultural monitoring and modeling. Poortinga et al. analyzed long-term land cover 
estimates for Vietnam, together with national agricultural yield statistics, to suggest significant increases in agricultural yield (per unit area) with relatively little change in total cropland area. Tiwari et al. presented methodologies using Earth Engine and satellite data to map irrigated and rain-fed wheat in Afghanistan, providing a tool for crop area estimation critical for yield forecasting and food security planning in remote regions. Analyses of this type can contribute significantly to understanding the spatial and temporal dimensions of land use and food insecurity in low income and often remote rural communities.

Three studies focused on rangeland monitoring. Ndungu et al. outline a comprehensive EO tool for rangeland monitoring tailored to stakeholder needs at local and regional scales in East Africa. The Rangeland Decision Support Tool provides easy access to satellite-based vegetation indices that can be integrated with ancillary GIS datasets specific to the needs of local communities and range managers. Anchang et al. developed a work-flow for mapping woody canopy cover using Google Earth Engine, local field data and expertise, machine learning, and data from the Sentinel satellites for Senegal, West Africa. The cloud-based computing approach reduces the computational barriers often faced by the EO community in countries with relatively poor internet bandwidth, and is a tool available for local calibration and application in other regions. Meanwhile, Ouko et al. demonstrated an approach to mapping and modeling invasive plants in the ecologically, economically, and culturally valuable rangelands of East Africa, using cell-phone based citizen scientist inputs on invasive plant locations, satellite, and GIS data, to map the presence and spread of invasive species. This study focused on an invasive shrub (Acacia reficiens) and prickly pear (Opuntia spp.), but similar approaches could be adopted in other regions where invasive plants are impacting ecosystem integrity and rural livelihoods.

\section{REFERENCES}

Brachet, G. (2004). From initial ideas to a European plan: GMES as an exemplar of European space strategy. Space Policy 20, 7-15. doi: 10.1016/j.spacepol.2003.11.002

Brown, M. E., Escobar, V. M., Aschbacher, J., Milagro-Pérez, M. P., Doorn, B., Macauley, M. K., et al. (2013). Policy for robust spacebased earth science, technology and applications. Space Policy 29, 76-82. doi: 10.1016/j.spacepol.2012.11.007

Friedl, L. (2017). "Chapter 4: Benefits assessment of applied earth science," in Satellite Earth Observations and Their Impact on Society and Policy, eds M. Onoda and O. Young (Singapore: Springer), 73-79. doi: 10.1007/978-981-10-3713-9_4

Kumar, S. A., Camacho, S., Searby, N. D., Teuben, J., and Balogh, W. (2020). Coordinated capacity development to maximize the contributions of space science, technology, and its applications in support of implementing global sustainable development agendas-a conceptual framework. Space Policy 51:101346. doi: 10.1016/j.spacepol.2019.1 01346
One paper in this Special Topic, by Flores-Anderson et al. examined the critical, but less well-known, potential to use hyperspectral imagery for lake water quality assessment. While the review paper by Leibrand et al. examines how satellite data can contribute to all aspects of planning and management of rural electrification, renewable and sustainable energy planning and management.

\section{CONCLUSION}

The studies in this Special Topic share a major common themethe use of EO to facilitate improved decision making by land users and managers at local, national, and regional scales. The value of $\mathrm{EO}$ for decision making in land cover monitoring, agricultural assessments, or streamflow predictions is high, given the spatial continuity, and the temporal return frequency afforded by space-based platforms. That value is particularly critical in developing countries where EO information is sometimes a key source for decision makers. Responsiveness to the specific needs of different stakeholders, based on local economic, cultural, and environmental conditions is especially important, because EO applications then have a higher chance of achieving real impact for local land users or via sustainable policy interventions. The result is a diversity of individual strands (approaches) to EO-informed solutions and it is up to scientific communities, such as SERVIR, to weave those solutions together in the hope and anticipation that these approaches will be replicated in other parts of the world. The scalability and replicability of activities in a global program such as SERVIR is critically dependent on these individual efforts. This compilation of articles provides greater insight into some of those activities.

\section{AUTHOR CONTRIBUTIONS}

All authors contributed equally to this article.

National Academies of Sciences, Engineering, and Medicine (2019). Thriving on Our Changing Planet: A Decadal Strategy for Earth Observation from Space. Washington, DC: The National Academies Press. doi: 10.17226/24938

Zell, E., Huff, A. K., Carpenter, A. T., and Friedl, L. A. (2012). A user-driven approach to determining critical earth observation priorities for societal benefit. IEEE Journal of Selected Topics in Applied Earth Observations and Remote Sensing, 5, 1594-1602. doi: 10.1109/JSTARS.2012.2199467

Conflict of Interest: The authors declare that the research was conducted in the absence of any commercial or financial relationships that could be construed as a potential conflict of interest.

Copyright (c) 2020 Hanan, Limaye and Irwin. This is an open-access article distributed under the terms of the Creative Commons Attribution License (CC BY). The use, distribution or reproduction in other forums is permitted, provided the original author(s) and the copyright owner(s) are credited and that the original publication in this journal is cited, in accordance with accepted academic practice. No use, distribution or reproduction is permitted which does not comply with these terms. 